\title{
Prescribing pre-exposure prophylaxis in general practice
}

Osama Bhatti, Darren So, Muhammad Abdullah Zahid, Vincent J Cornelisse

\section{Background}

Pre-exposure prophylaxis (PrEP) is a highly effective method of human immunodeficiency virus (HIV) prevention available to prescribe on the Pharmaceutical Benefits Scheme general schedule.

\section{Objective}

The aim of this article is to provide information and guidance to general practitioners (GPs) and other healthcare providers on providing PrEP, and to discuss efficacy, adverse effects, monitoring and further resources.

\section{Discussion}

GPs can play a central role in discussing HIV prevention strategies for people who may be at risk of HIV. An open and engaging discussion will assist patients in making an informed decision on whether PrEP is suitable for them.
HUMAN IMMUNODEFICIENCY VIRUS (HIV) remains a global health concern, with an estimated 38 million people living with HIV worldwide and 690,000 acquired immunodeficiency syndrome-related deaths in 2019. ${ }^{1}$ In Australia, 28,918 people were living with HIV in $2019 .^{2}$

Gay, bisexual and other men who have sex with men (GBMSM) remain the population most at risk of HIV in Australia, accounting for 597 of the 903 new notifications in $2019 .{ }^{2}$ In the 2020 Gay Community Period Survey, 8.1\% of GBMSM reported an HIV-positive test result $(92.1 \%$ of whom were on antiretroviral treatment, and $93.4 \%$ having an undetectable viral load). ${ }^{3}$

Among people who inject drugs (PWID) and attend needle and syringe program sites, HIV prevalence in 2019 was estimated to be $2.3 \%$ overall. Within this group, the highest prevalence was among GBMSM who inject drugs, at a very high $32 \%{ }^{4}$

In Australia, an estimated 10.5\% of people living with HIV remain undiagnosed. ${ }^{2}$ General practitioners are in a prime position to identify these individuals by screening patients who may be at risk. When taking a medical history in general practice, questions on sexual relationships and sexual practices should be included routinely for all patients. This allows for proactive identification of HIV risk factors, which should then prompt
HIV testing and discussion about risk reduction strategies, including condom use, immunisations (hepatitis A and B, and human papillomavirus) and HIV pre-exposure prophylaxis (PrEP).

\section{What is HIV PrEP?}

PrEP involves the use of HIV medications (co-formulated tenofovir disoproxil with emtricitabine [TD/FTC]) by people who are HIV negative to reduce their risk of HIV from potential exposure in the near future. PrEP was listed on the Pharmaceutical Benefits Scheme (PBS) general schedule in 2018, allowing prescription by any medical practitioner or sexual health nurse practitioner in Australia. ${ }^{5}$

The approved formulations currently available on the PBS general schedule for PrEP are:

- tenofovir disoproxil fumarate $300 \mathrm{mg}+$ emtricitabine $200 \mathrm{mg}$

- tenofovir disoproxil maleate $300 \mathrm{mg}+$ emtricitabine $200 \mathrm{mg}$

- tenofovir disoproxil phosphate $291 \mathrm{mg}+$ emtricitabine $200 \mathrm{mg}$.

Further options that are being investigated for PrEP, but not yet available in Australia at the time of writing, include co-formulated tenofovir alafenamide and emtricitabine (which may confer a safer renal/bone mineral density profile), ${ }^{6}$ and cabotegravir (a long-acting injectable form of PrEP). ${ }^{7}$ 


\section{Efficacy}

When taken once daily, PrEP can reduce the risk of HIV transmission by up to $99 \%$ from sexual transmission, ${ }^{8,9}$ and up to $74 \%$ from intravenous drug use. ${ }^{10}$

One of the largest randomised trials of PrEP was the Preexposure Prophylaxis Initiative (iPrEx) study (2007-11), ${ }^{11} \mathrm{a}$ multinational, randomised, placebocontrolled trial of the effectiveness of daily TD/FTC to reduce the risk of HIV among 2499 HIV-negative participants, mainly GBMSM and a small number of transgender women. Results indicated that participants with a detectable plasma level of study medication had a relative risk reduction of $92 \%$ ( $95 \%$ confidence interval $[\mathrm{CI}]:$ 40, 99, $P<0.001) .{ }^{11}$ A pharmacokinetic analysis of the $\mathrm{iPrEx}$ data indicated a risk reduction of $96 \%$ for participants with study drug levels indicating four doses per week $(95 \% \mathrm{CI}$ : $90,>99 \%)$ and $99 \%$ for seven doses per week (99\% CI: $96,>99 \%){ }^{12}$

Similar high protective levels were seen in the open-label UK PRe-exposure Option for reducing HIV in the UK: immediate or Deferred (PROUD) study, ${ }^{13}$ which randomised 544 HIV-negative GBMSM to receive daily TD/FTC either immediately or after a deferral period of one year. Three HIV infections occurred in the immediate-start group receiving PrEP compared with 20 in the deferred group (relative risk reduction: $86 \%, 90 \%$ CI: 64 , $96, P<0.0001)$. It is important to note that none of the three HIV-positive patients in the immediate-start arm were taking PrEP at the time of HIV seroconversion, based on records of clinical attendance and prescription history, suggesting higher efficacy than was reported in the intention-to-treat analysis. ${ }^{13}$

The Partners PrEP Study assessed the efficacy of PrEP among heterosexual couples, and indicated a relative risk reduction of $75 \%$ in HIV transmission (95\% CI: $55,87, P<0.001$ ), which increased to $90 \%$ among participants with a detectable tenofovir disoproxil fumarate plasma level. ${ }^{14}$ This study only assessed recent, rather than consistent, PrEP use, and it is likely that the consistent daily use of PrEP would be just as efficacious for heterosexual men and women as for GBMSM.
The Bangkok Tenofovir Study ${ }^{10}$ reported on the efficacy of PrEP in reducing HIV transmission among PWID across 17 drug-treatment clinics in Bangkok by up to $74 \%$. However, that study may be of limited relevance to the Australian context, where PWID have easier access to needle-sharing programs and treatment facilities, and therefore, a lower risk of HIV than in Bangkok.

Patients who elect to take PrEP should be counselled on the importance of medication adherence, barrier protection against other sexually transmissible infections (STIs) and review appointments every three months (refer to Monitoring).

\section{Indications for PrEP}

Guidelines from the Australasian Society for HIV, Viral Hepatitis and Sexual Health Medicine (ASHM) recommend PrEP for patients who disclose recent, or who anticipate future, risk of HIV acquisition, and for patients whose quality of life will improve with PrEP. ${ }^{15}$

For GBMSM, a high risk of HIV may be indicated by a history of condomless anal intercourse with casual partners, engaging in sexualised drug use ('chemsex' agents, such as crystal methamphetamines, ecstasy, gamma-hydroxybutyrate and cocaine) or recent diagnoses of syphilis, rectal gonorrhoea, or rectal chlamydia. ${ }^{15-17}$ However, GBMSM who do not identify these particular risk factors may nonetheless be at risk of HIV and require PrEP. ${ }^{15,17}$

People who engage in condomless sex with HIV-positive partners may benefit from PrEP if their partner is not on effective treatment. ${ }^{15,16} \mathrm{HIV}$-positive individuals on treatment and with an undetectable viral load cannot transmit HIV sexually. ${ }^{18}$ This principle is also known as 'undetectable =untransmissible'. Therefore, if a person's only potential HIV exposure is through sexual contact with someone who has an undetectable viral load, then that person may not require PrEP. However, clinicians must bear in mind that this person may still require PrEP if they anticipate sexual contact with other partners.

Trans and gender-diverse people are also eligible to access PrEP to reduce their
HIV risk. Unfortunately, trans and genderdiverse people have not been adequately represented in PrEP trials, so the efficacy of PrEP in this population currently needs to be extrapolated from other trial populations. ${ }^{15}$

PrEP may also be an important HIV-reduction strategy for PWID, particularly if they inject with people who are HIV-positive, GBMSM or are otherwise at risk due to their sexual practices. ${ }^{15,16}$

An open and engaging history with the patient will help the prescriber evaluate the need for PrEP on an individualised basis. ${ }^{15}$ It is important to note that some people may be reluctant to disclose their HIV risk to a provider due to the fear of stigma. For this reason, the ASHM guidelines recommend that anyone who requests PrEP should be assisted with accessing PrEP. ${ }^{15}$ Further information regarding PrEP suitability criteria is available through the ASHM guidelines or by contacting a sexual health physician (refer to Resources).

\section{Clinical criteria}

Patients who are suitable for starting PrEP will need a negative HIV test within the past four weeks (or evidence that a current HIV test is pending). ${ }^{5}$ If there has been a recent HIV exposure within the preceding 72 hours, patients should instead be considered for non-occupational post-exposure prophylaxis (nPEP). ${ }^{15,16}$ This may consist of referral to a nearby nPEP service, or if such a service is not conveniently available, these patients should be offered rapidly initiated PrEP, which involves taking the first PrEP pill and performing an HIV test on the day of presentation. These cases should be discussed with either the local state-based PEP hotline (www.getpep.info) or with an HIV physician. If the recent HIV exposure was outside the 72-hour window period for nPEP, patients may commence PrEP with monitoring for seroconversion for the next 2-8 weeks before commencing routine monitoring (for further information, refer to the ASHM decision-making tool web link under in Resources). ${ }^{15}$

It is now common practice to prescribe PrEP at the same visit that the initial 
HIV test is taken, because delaying the prescription of PrEP can result in ongoing HIV risk.

Tenofovir must not be prescribed for patients with a renal function of $<60 \mathrm{~mL} / \mathrm{min} / 1.73 \mathrm{~m}^{2} \cdot{ }^{15,16} \mathrm{PrEP}$ may be prescribed for women who are at risk of pregnancy or are pregnant and who are at risk of HIV, ${ }^{15}$ such as those with HIV-positive partners who are not taking antiviral medications or are not virally suppressed. ${ }^{15}$ Patients with chronic hepatitis B may take daily PrEP, but need to be advised that cessation of PrEP may trigger a flare of hepatitis B, and therefore, they may require close medical supervision after the cessation of PrEP. ${ }^{15,16}$ They must also be warned that intermittent dosing of PrEP is contraindicated, as it risks inducing antiviral resistance in their hepatitis B virus. ${ }^{15,16}$

\section{How to take PrEP}

Daily PrEP involves taking a co-formulated tablet of TD/FTC once a day. Most people are advised to take seven days of PrEP before they achieve protective medication levels, whereas GBMSM can take two tablets (a double dose) 2-24 hours before intercourse, before then continuing with one tablet per day. ${ }^{15}$

A second method of taking PrEP is 'on-demand PrEP' (or 'event-driven PrEP'). This method is recommended only for cis-gender GBMSM, or trans or gender-diverse people whose HIV risk is exclusively the result of receptive anal intercourse. On-demand PrEP involves the 2-1-1 rule: two tablets taken 2-24 hours before sex, one tablet taken 24 hours after the first dose and one tablet taken 48 hours after the first dose. ${ }^{19}$ Findings from the Intervention Préventive de l'Exposition aux Risques avec et pour les Gays (IPERGAY) trial reported an $86 \%$ reduction in HIV incidence using this on-demand regimen, ${ }^{20}$ and this risk reduction increased to $97 \%$ in the IPERGAY open-label extension. ${ }^{21}$ This method may suit GBMSM who are having sex less than twice a week and are able to delay sex for two hours to allow the loading dose to become effective. ${ }^{15}$ On-demand PrEP is not recommended for heterosexual males and females, as there have been no successful studies on on-demand PrEP in these populations. On-demand PrEP is also not recommended for people with chronic hepatitis B infection, due to the risk of antiviral resistance in their hepatitis B virus. ${ }^{15}$

\section{Adverse effects}

PrEP is generally well tolerated; however, patients should be made aware of a possible 'start-up syndrome', where side effects such as nausea, gastrointestinal upset, flatulence and headaches, may occur in the first month. ${ }^{15}$ PrEP may also lead to unintentional weight loss of around $5 \%$ of body weight. ${ }^{11}$

Tenofovir disoproxil carries a known, but uncommon, risk of nephrotoxicity in HIV treatment trials of about one in 1000 person-years. ${ }^{22}$ In the iPrEx trial, seven of 1251 patients taking $\operatorname{PrEP}(0.5 \%)$ developed persistently elevated creatinine levels during PrEP use, requiring discontinuation. All elevations in serum creatinine resolved within 4-20 weeks upon cessation of PrEP. ${ }^{7}$

PrEP may also lead to a slight decrease in bone mineral density (BMD) by 1-2\%. However, upon cessation, participants' $\mathrm{BMD}$ returned to baseline within 12-18 months. ${ }^{23}$ Routine assessment of BMD during PrEP use is not considered necessary. ${ }^{16}$

\section{Monitoring}

Patients who are on PrEP will need regular reviews with their doctor every three months, and this should be facilitated by the use of follow-up reminder mechanisms. These follow-up visits should involve checking for medication adherence, adverse effects, ongoing need for PrEP, prescribing a three-month supply of PrEP and organising the appropriate monitoring tests.

At baseline, patients should have a recent negative HIV test (preferably within the past week, but at least within the past four weeks of starting PrEP), 5,15 or have an HIV test performed on the day of starting PrEP, even if the result may not yet be available. ${ }^{5}$ Other baseline tests include hepatitis B and C serology, and assessment of kidney function via estimated glomerular filtration rate (eGFR) and urine protein:creatinine ratio (not an albumin:creatinine ratio), screening for other STIs (as per Australian STI guidelines) and a pregnancy test for women of child-bearing potential. ${ }^{15}$

After initiating PrEP, patients will need three-monthly HIV testing, as prolonged exposure to TD/FTC following undiagnosed HIV infection may lead to antiretroviral resistance. ${ }^{15,16}$ In addition, patients should be offered STI testing appropriate to their practices, and these may include tests, such as chlamydia and gonorrhoea testing (on first-pass urine, anorectal swabs and oropharyngeal swabs), syphilis serology and hepatitis C serology (checked at least annually). ${ }^{24}$

Renal function (eGFR and urine protein:creatinine ratio) should be performed at baseline, at three months, and then every six months thereafter. Some patients may require more frequent (ie every three months) renal function testing, such as those aged $<25$ years or $>40$ years, those with a baseline eGFR $<90 \mathrm{~mL} / \mathrm{min} / 1.73 \mathrm{~m}^{2}$ or those with additional risk factors for renal disease, such as hypertension or diabetes. ${ }^{15}$

\section{Cessation}

Patients may decide to cease PrEP when they are no longer at risk of HIV. Cis-gender GBMSM may stop PrEP safely by taking PrEP for two consecutive days (at 24 and 48 hours) after their last sexual exposure, whereas others will need to continue PrEP for 28 days after their last exposure. ${ }^{15}$ Clinicians should document clients' HIV status and reason for discontinuation, and check for recent medication adherence and reported sexual behaviours at the time of discontinuation. ${ }^{15,16}$ Clinicians should also explore with their patients how they may safely re-commence PrEP if their HIV risk increases again, and ideally this is discussed at the time of considering cessation of PrEP. Patients with chronic hepatitis B infection should exercise caution when ceasing PrEP, as it may result in a flare of hepatitis $B$ virus. ${ }^{15,16}$ 
These patients may require monitoring of their liver function tests in the period after stopping PrEP, and ideally this is discussed with their hepatologist. All patients ceasing PrEP should be reminded to use condoms for protection, seek regular sexual health checks and discuss restarting PrEP in the future if needed.

\section{Conclusion}

The provision of PrEP provides an opportunity to engage with young people, GBMSM, trans and gender-diverse people, PWID and other people who are at risk of HIV. These patient populations often find it difficult to engage with medical services, and therefore, PrEP may provide a gateway to foster comprehensive care.

PrEP is now more accessible due to generic options available at most pharmacies, at similar costs for both Medicare holders and those with private prescriptions. Patients for whom cost is a barrier may import PrEP from trusted online pharmacies (such as those listed on www.pan.org.au), as permitted by the Therapeutic Goods Administration's personal importation scheme.

\section{Resources}

- Australasian Society for HIV, Viral Hepatitis and Sexual Health Medicine (ASHM) website:

- Brief Decision making in PrEP resource for clinicians, https://ashm. org.au/resources/hiv-resources-list/ decision-making-in-prep

- Brief PrEP dosing guide for clinicians, https://ashm.org.au/ resources/hiv-resources-list/ prep-dosing-guide

- PrEP patient information, https://ashm.org.au/resources/ hiv-resources-list/prep-patientinformation

- Full PrEP guidelines, https:// prepguidelines.com.au

- nPEP hotline, www.getpep.info

\section{Authors}

Osama Bhatti MBBS, FRACGP, General Practitioner, Monash University Health Services, Monash University, Vic
Darren So MBBS, FRACGP, General Practitioner, Monash University Health Services, Monash University, Vic

Muhammad Abdullah Zahid MBBS, Junior Medical Officer, Army Medical College, Rawalpindi, Pakistan Vincent J Cornelisse BSc (Hons), MBBS, FRACGP, FAChSHM, PhD, Staff Specialist in sexual health medicine, Kirketon Road Centre, NSW Health, NSW; Conjoint Senior Lecturer, The Kirby Institute, UNSW, NSW

Competing interests: VJC reports personal speaker fees from Gilead Sciences and advisory board personal fees from ViiV Healthcare, outside of the submitted work.

Funding: None.

Provenance and peer review: Not commissioned, externally peer reviewed.

Correspondence to:

oibhatti@gmail.com

\section{References}

1. UNAIDS. UNAIDS Data 2019. Geneva, $\mathrm{CH}$ : UNAIDS, 2019.

2. Kirby Institute. HIV. Sydney, NSW: UNSW, 2020 Available at https://data.kirby.unsw.edu.au/hiv [Accessed 10 April 2021].

3. Chan C, Broady T, Bavinton B, et al. Gay community periodic survey: Sydney 2020. Sydney, NSW: UNSW Sydney, 2020.

4. Heard S, Iversen J, Geddes L, Maher L. Australian NSP survey: Prevalence of HIV, HCV and injecting and sexual behaviour among NSP attendees, 25-year National Data Report 1995-2019. Sydney, NSW: The Kirby Institute, UNSW Sydney, 2020.

5. Department of Health. PBS and RPBS section 85 date of supply data. Canberra, ACT: DoH, 2021. Available at www.pbs.gov.au/info/statistics/dosand-dop/dos-and-dop [Accessed 12 April 2021].

6. Mayer KH, Molina JM, Thompson MA, et al. Emtricitabine and tenofovir alafenamide vs emtricitabine and tenofovir disoproxil fumarate for HIV pre-exposure prophylaxis (DISCOVER): Primary results from a randomised, double-blind, multicentre, active-controlled, phase 3, noninferiority trial. Lancet 2020;396(10246):239-54. doi: 10.1016/S0140-6736(20)31065-5.

7. Kirby T. Cabotegravir, a new option for PrEP. Lancet Infect Dis 2020;20(7):781. doi: 10.1016/ S1473-3099(20)30497-7.

8. Grant RM, Anderson PL, McMahan V, et al. Uptake of pre-exposure prophylaxis, sexual practices, and HIV incidence in men and transgender women who have sex with men: A cohort study. Lancet Infect Dis 2014;14(9):820-29. doi: 10.1016/S14733099(14)70847-3.

9. Centers for Disease Control and Prevention. Effectiveness of prevention strategies to reduce the risk of acquiring or transmitting HIV. Atlanta, GA: CDC, 2019. Available at www.cdc.gov/ hiv/risk/estimates/preventionstrategies.html [Accessed 12 April 2021].

10. Choopanya K, Martin M, Suntharasamai P, et al. Antiretroviral prophylaxis for HIV infection in injecting drug users in Bangkok, Thailand (the Bangkok Tenofovir Study): A randomised, doubleblind, placebo-controlled phase 3 trial. Lancet 2013;381(9883):2083-90. doi: 10.1016/S01406736(13)61127-7.

11. Grant RM, Lama JR, Anderson PL, et al. Preexposure chemoprophylaxis for HIV prevention in men who have sex with men. N Engl J Med 2010;363(27):2587-99. doi: 10.1056/ NEJMoa1011205.
12. Anderson PL, Glidden DV, Liu A, et al. Emtricitabine-tenofovir concentrations and preexposure prophylaxis efficacy in men who have sex with men. Sci Transl Med 2012;4(151):151ra125. doi: 10.1126/scitranslmed.3004006.

13. McCormack S, Dunn DT, Desai M, et al. Preexposure prophylaxis to prevent the acquisition of HIV-1 infection (PROUD): Effectiveness results from the pilot phase of a pragmatic open-label randomised trial. Lancet 2016;387(10013):53-60. doi: 10.1016/S0140-6736(15)00056-2.

14. Baeten JM, Donnell D, Ndase P, et al. Antiretroviral prophylaxis for HIV prevention in heterosexual men and women. N Engl J Med 2012;367(5):399-410. doi: 10.1056/NEJMoa1108524.

15. The Australasian Society of HIV, Viral Hepatitis and Sexual Health Medicine (ASHM) PrEP guidelines update. Prevent HIV by prescribing PrEP. Sydney, NSW: ASHM, 2019.

16. Centers for Disease Control and Prevention. US public health service: Preexposure prophylaxis for the prevention of HIV infection in the United States - 2017 update: A clinical practice guideline. Atlanta, GA: CDC, 2018.

17. Cornelisse VJ, Fairley CK, Stoove M, et al. Evaluation of preexposure (PrEP) eligibility criteria, using sexually transmissible infections as markers of human immunodeficiency virus (HIV) risk at enrollment in PrEPX, a large Australian HIV PrEP trial. Clin Infect Dis 2018;67(12):1847-52. doi: 10.1093/cid/ciy370.

18. Bavinton BR, Pinto AN, Phanuphak N, et al. Viral suppression and HIV transmission in serodiscordant male couples: An international, prospective, observational, cohort study. Lancet HIV 2018;5(8):e438-47. doi: 10.1016/S23523018(18)30132-2.

19. World Health Organization. What's the $2+1+1$ ? Event-driven oral pre-exposure prophylaxis to prevent HIV for men who have sex with men: Update to WHO's recommendation on oral PrEP. Geneva, CH: WHO, 2019.

20. Molina JM, Capitant C, Spire B, et al. On-demand preexposure prophylaxis in men at high risk for HIV-1 infection. N Engl J Med 2015;373(23):2237-46. doi: 10.1056/ NEJMoa1506273.

21. Molina JM, Charreau I, Spire B, et al. Efficacy, safety, and effect on sexual behaviour of on-demand pre-exposure prophylaxis for HIV in men who have sex with men: An observational cohort study. Lancet HIV 2017;4(9):e402-10. doi: 10.1016/S2352-3018(17)30089-9.

22. Medland NA, Chow EP, Walker RG, Chen M, Read TR, Fairley CK. Incidence of renal Fanconi syndrome in patients taking antiretroviral therapy including tenofovir disoproxil fumarate. Int J STD AIDS 2018;29(3):227-36. doi: 10.1177/0956462417722133.

23. Glidden DV, Mulligan K, McMahan V, et al. Brief report: Recovery of bone mineral density after discontinuation of tenofovir-based HIV pre-exposure prophylaxis. J Acquir Immune Defic Syndr 2017;76(2):177-82. doi: 10.1097/ QAI.0000000000001475.

24. Cornelisse VJ, Traeger MW, Wright EJ, et al. Low incidence of hepatitis $C$ among a cohort of HIV-negative gay and bisexual men using HIV pre-exposure prophylaxis (PrEP) in Melbourne, Australia, and the contribution of sexual transmission. J Acquir Immune Defic Syndr 2021. doi: 10.1097/QAI.0000000000002685.

correspondence ajgp@racgp.org.au 